[CONTRIBUtion From THH LABORATORY OF THE UNIVERSITY OF MiNnESOTA.]

\title{
PREPARATION OF 2.5-DIMETHYLBENZALDEHYDE; THE ESTABLISHMENT OF ITS CONSTITUTION AND PREP- ARATION OF SOME OF ITS DERIVATIVES.
}

By Everhart P. Harding and Lillian CoheN.

Received July 5, xgor.

$F^{E W}$ compounds have offered a more interesting study than the alone but to their utilitarian value as well. Gattermann ${ }^{1}$ says that in all known aldehydes (containing one or more methyl groups in the benzene ring) prepared by the Gattermann-Koch method, ${ }^{1}$ the aldehyde group always occupies the $p$-position relative to a methyl group." The object of this paper was to determine whether the aldehyde could enter the benzene ring without occupying a $p$-position to any of the methyl groups which resulted in the preparation of 2.5-dimethylbenzaldehyde, the establishment of its constitution and the preparation of some of its derivatives.

PREPARATION OF 2.5-DIMETHYLBENZALDEHYDE.

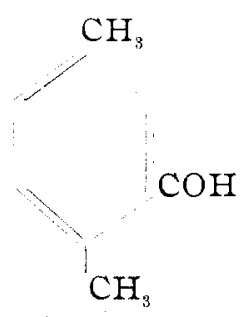

2.5-Dimethylbenzaldehyde was prepared according to the Gattermann-Koch method, ${ }^{3}$ which depends upon the action of the hypothetical formyl chloride upon aromatic hydrocarbons in the presence of aluminum and cuprous chlorides. The temperature of the xylene mixture was kept at $40^{\circ}$ and the time required for the thickening of the mass varied from five to ten hours, depending upon the quality of the aluminum chloride used. The double compound of aldehyde and aluminum chloride was decomposed by transferring to a flask containing ice and the aldehyde and

1 Ber. d. chem., Ges. 30, 1622.

2 Ibid., 30, 1622; Wilhelm Graf: Inaug. Dissertation, I 899 , Tiniverstäts Buchdruckerei von J. Horning, Heidelberg; J.prakt. Chem. Neue Folg., 62, 1900.

3 Ber. d. chem. Ges. 30, 2622 , 
unchanged xylene removed by steam distillation and taken up with ether. After removing the ether by distillation, the residue was subjected to repeated fractional distillation. The aldehyde distilled over at $219^{\circ}-229^{\circ}$ (uncorr.) as a colorless liquid which quickly turned to light yellow, due to its ready oxidation.

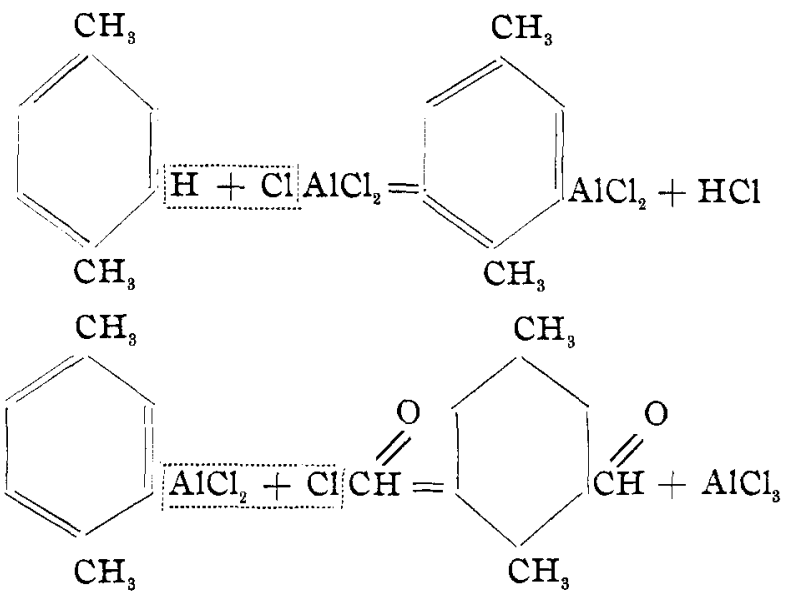

The aldehyde does not form an addition product with a saturated solution of sodium bisulphite ${ }^{1}$ which makes the usual method of purifying the aldehydes by their conversion into the corresponding bisulphite compounds impracticable. The aldehyde was dissolved in very weak alcohol and then shaken for two days in a shaking machine with a saturated solution of pure sodium bisulphite dissolved in water.

The analysis gave:

0.1400 gram of the substance burned with cupric oxide gave 0.4140 gram carbon dioxide and 0.0933 gram water, equivalent to $0.1129 \mathrm{gram}$ carbon and 0.0140 gram hydrogen.

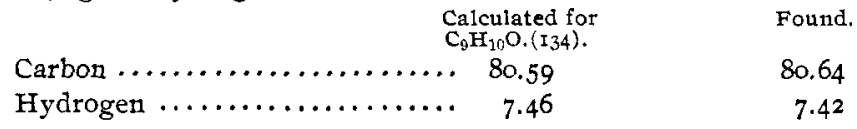

2.5-Dimethylbenzaldehyde is a colorless liquid with the characteristic benzaldehyde odor. It oxidizes very readily. Upon distillation a residue remains which, upon cooling, crystallizes in colorless needles. On exposure to the air it changes to a light yellow color and subsequently to prismatic needles which melt at tried.

1 The yellow commercial sodium bisulphite was not obtainable and consequently not 
the melting-point of the corresponding acid. ${ }^{1}$ It oxidizes so readily that upon distillation, air must be excluded from the apparatus and the distillate quickly cooled in ice-water. It is soluble in all the common solvetits except water.

PREPARATION OF 2.5-DIMETHYLBENZOIC ACID.

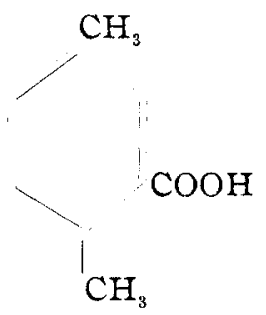

By the oxidation of 2.5-dimethylbenzaldehyde to the corresponding acid the position assumed by the aldehyde group in the benzene ring and consequently the constitution of the aldehyde was determined.

Since chromic acid and potassium permanganate oxidize the methyl group as well as the aldehyde group a strong solution of sodium hydroxide was used. Two grams of 2.5 dimethylbenzaldehyde and a cold solution of I.8 grams of sodium hydroxide dissolved in 1.5 grams water were placed in a well-stoppered bottle. The bottle was placed in a shaking machine and shaken until a permanent emulsion was formed. After standing for several days this hardened to a yellow solid. The corresponding alcohol and the sodium salt of the corresponding acid were formed. The alcohol was removed by treating with water and extracting with ether. Upon acidifying the aqueous solution with dilute hydrochloric acid the 2.5-dimethylbenzoic acid separated as a white crystalline precipitate. Recrystallized from 50 per cent. alcohol it melted at $124^{\circ}-125^{\circ}$ (uncorr.). A 50 per cent. alcoholic solution was boiled with animal charcoal and filtered. The precipitate formed on cooling melted at $\mathrm{I} 24^{\circ}-\mathrm{I} 25^{\circ}$.

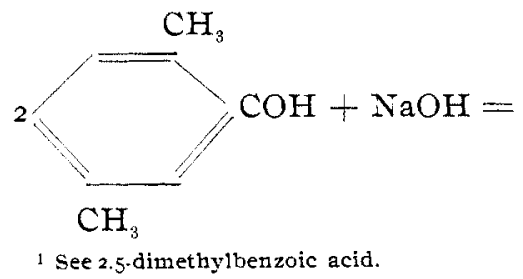


The analysis gave :

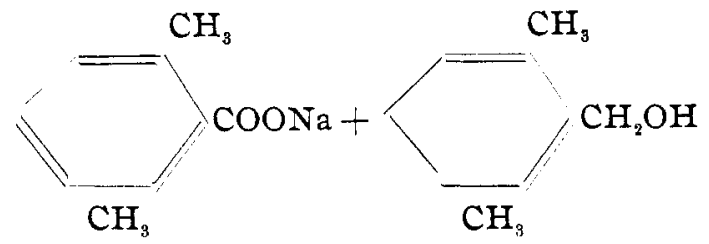

0.1006 gram burned with cupric oxide gave 0.2657 gram carbon dioxide and 0.0585 gram water equivalent to 0.07246 gram carbon and $0.0065 \mathrm{gram}$ hydrogen.

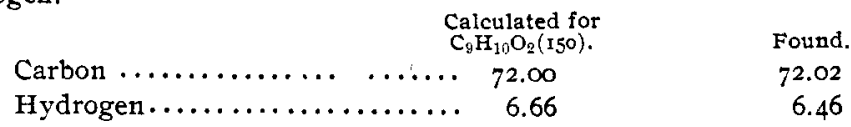

2.5-Dimethylbenzoic acid forms colorless needles of the monoor triclinic system. $\angle \beta=58^{\circ} 2 \mathrm{I}^{\prime}{ }^{1}{ }^{1}$

External angle measured on oro $=48^{\circ} 36^{\prime}$.

$$
\begin{aligned}
\infty a: b: \infty c & =\text { oIo }=\text { clinopinacoid. } \\
a: b: \infty c & =\text { II }=\text { prism. } \\
\infty a: \infty b: c=\text { OOI } & =\text { basal pinacoid. } \\
a: \infty b: \infty c & =\text { IOI }=\text { orthopinacoid. }
\end{aligned}
$$

These crystals are soluble in methyl alcohol, ethyl alcohol, toluene, xylene, benzene, chloroform, and acetone. They are insoluble in cold and hot water. When boiled with pure animal charcoal and 50 per cent. alcohol, the filtrate turns slightly blue; the same blue color is observed during combustion.

If the aldehyde group always occupied the para position relative to a methyl group and if it were possible for the aldehyde group to exchange positions with one of the methyl groups, one of four acids of the corresponding aldehydes, viz., $p$-tolyl acetaldehyde, $2.4^{-}, 3.4^{-}$, or $2.5^{-d i m e t h y l b e n z o i c ~ a c i d, ~ m i g h t ~ h a v e ~}$ formed.

Paratolylacetic acid oxidizes with chromic acid to terephthalic acid and has a lower melting-point than that found. ${ }^{2}$ 2.4- and 3.4dimethylbenzoic acids also have different melting-points and different physical properties. Jacobson ${ }^{3}$ prepared an acid, ${ }^{4}$ which he called $p$-xylic acid, by the action of monobromparaxylene upon amalgam and phosgene and which melted at $132^{\circ}$. He also obtained a $p$-xylic acid which melted at $163^{\circ}$. Fittig and

${ }^{1}$ Kindly determined by Dr. Berkey.

2 Ber. $d$ chem. Ges., I5, 17444; 42, 1882 .

3 Ibid., I888, p. 1933: 22, 1230; 20, 2051; 18, 1281.

4 Ibid., 14, $2111 ; 17,2374$. 
Laubinger ${ }^{1}$ prepared an acid by oxidizing pseudocumene with dilute nitric acid which melted at $163^{\circ}$, and which they called p-xylic acid.

In all probability the so-called $p \cdot x y-1 i c$ acid melting at $I 63^{\circ}$ obtained by Jacobson, and the acid obtained by Laubinger and Fittig melting at $163^{\circ}$, are the orthoxylic acid recently obtained by Graf ${ }^{2}$ by treating orthoxylic aldehyde with strong potassium hydroxide and which melted at $163^{\circ}$. A further proof that the acid formed is neither 2.4-nor 3.4-dimethylbenzoic acid is shown in this paper by the comparison of the melting-points of their corresponding cinnamic acids.

This proves that the aldehyde group does not occupy a para position relative to one of the methyl groups but a meta position to one and an ortho position to the other group.

ALDAZINE OF 2.5-DIMETHYLBENZALDEHYDE.

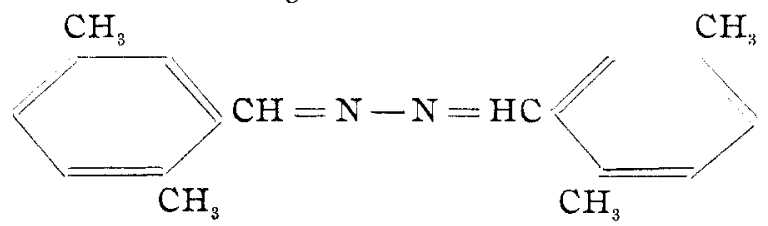

The aldazine of 2.5-dimethylbenzaldehyde was prepared according to the method of Curtius and Jay." Five grams of aldehyde dissolved in dilute alcohol was added to a solution of 2.5 grams hydrazine sulphate dissolved in an excess of water. By violent shaking in a shaking machine a light yellow precipitate formed which, when filtered off, washed consecutively with water and cold alcohol, and recrystallized from hot alcohol, melted at $114^{\circ}-114.5^{\circ}$.

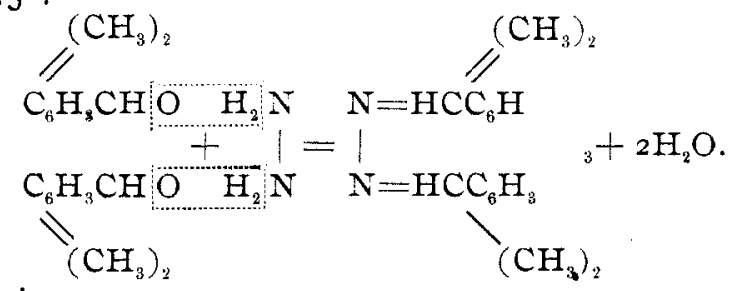

The analysis was:

0.IOgI gram burned with cupric oxide gave at $24^{\circ}$ and $74 \mathrm{I} \mathrm{mm} ., 10.8 \mathrm{cc}$. saturated nitrogen gas equivalent to $11.799 \mathrm{mg}$. nitrogen.

I Anx. Chem. (Liebig), 151, 273 .

2 Wilhelm Graf: Inaug. Dissertation, r899, Universtäts Buchdruckerei von J.Hörning, Heidelberg.

37.prakt. Chem., Neue Folge, $8 \mathbf{9}, 43$. 


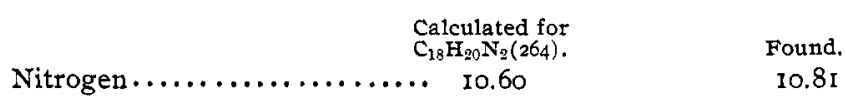

The aldazine of 2.5-dimethylbenzaldehyde is soluble in methyl and ethyl alcohols, ether, benzene, toluene, xylene, and glacial acetic acid. It is insoluble in water.

PHENYLHYDRAZONE OF 2,5-DIMETHYLBENZALDEHYDE.

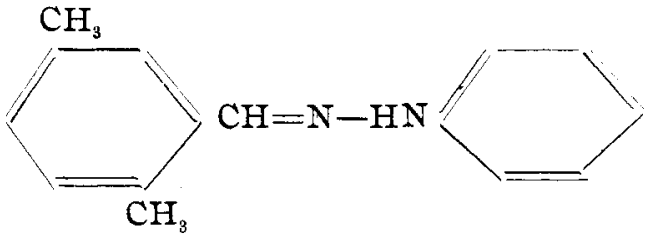

Five grams of 2.5-dimethylbenzaldehyde dissolved in an equal weight of alcohol were added to a mixture of 1.8 grams of phenylhydrazine dissolved in a mixture of two grams of glacial acetic acid and an equal volume of water. Upon shaking, the hydrazone separated as a yellowish-white precipitate which, filtered, washed consecutively with water and cold alcohol, and recrystallized from glacial acetic acid, melted at $84^{\circ}-85.5^{\circ}$.

$\left(\mathrm{CH}_{3}\right)_{2}$

$\mathrm{C}_{6} \mathrm{H}_{3} \mathrm{CHO}+\mathrm{H}_{2} \mathrm{NNHC}_{6} \mathrm{H}_{5}=$

$$
\mathrm{H}_{2} \mathrm{O}+\frac{\left(\mathrm{CH}_{3}\right)_{2}}{\mathrm{C}_{6} \mathrm{H}_{3} \mathrm{CH}}=\mathrm{N}-\mathrm{NHC}_{6} \mathrm{H}_{5}
$$

It is necessary to conduct this experiment quickly, as the hydrazone is very unstable. The crystals soon turn a yellowish brown and subsequently a deep red. This red color appears when an alcoholic solution of the hydrazone stands for some time; also when the hydrazone is placed in an exhausted desiccator.

Analysis :

$0.054^{2}$ gram burned with copper oxide gave at $17^{\circ}$ and $750 \mathrm{~mm} ., 6 \mathrm{cc}$. of saturated nitrogen gas, equivalent to $6.870 \mathrm{mg}$. nitrogen.

$$
\begin{aligned}
& \text { Calculated for } \\
& \mathrm{C}_{15} \mathrm{H}_{16} \mathrm{~N}_{2}(224) \text {. Found } \\
& \text { Nitrogen................. } \quad 12.50 \\
& \text { I } 2.67
\end{aligned}
$$

Hydrazone of 2.5-dimethylbenzaldehyde is soluble in ethyl and methyl alcohols, ether, benzene, toluene, and xylene. It is insoluble in water. 
OXIME OF 2.5-DIMETHYLBENZALDEHYDE.

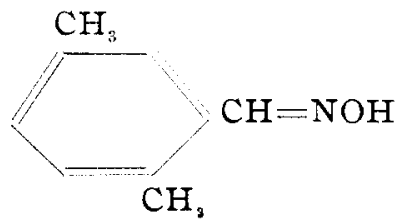

Two grams of 2.5-dimethylbenzaldehyde were dissolved in I5 grams of alcohol. To this solution was added a solution of I.8 grams of hydroxylamine hydrochloride in 5 grams of water and subsequently a solution of 4.5 grams of potassium hydroxide in 5 grams of water. The mixture was placed in a small roundbottomed flask, provided with a reflux condenser and heated for two hours upon a water-bath. The alcohol was then partially evaporated and the solution poured into 50 grams of water and filtered to remove any unchanged aldehyde. The free oxime was then precipitated by the addition of dilute hydrochloric acid. The colorless crystalline precipitate was filtered off, pressed upon an unglazed porcelain plate and recrystallized from 40 per cent. alcohol.

The crystals melted at $83.5^{\circ}-84^{\circ}$.

$$
\begin{array}{ll}
\left(\mathrm{CH}_{3}\right)_{2} & \left(\mathrm{CH}_{3}\right)_{2} \\
\mathrm{C}_{16} \mathrm{H}_{3} \mathrm{CHO}+\mathrm{H}_{2} \mathrm{NOH}= & \mathrm{C}_{6} \mathrm{H}_{3} \mathrm{CH}=\mathrm{NOH}+\mathrm{H}_{2} \mathrm{O}
\end{array}
$$

Analysis :

0.0939 gram burned with cupric oxide gave at $19^{\circ}$ and $744 \mathrm{~mm} ., 8 \mathrm{cc}$. saturated nitrogen gas, equivalent to $8.75^{2} \mathrm{mg}$. nitrogen.

Calculated for

$\mathrm{C}_{9} \mathrm{H}_{11} \mathrm{NO}$ (149).

Nitrogen.................. 9.39
Found.

$9 \cdot 32$

The oxime is soluble in ethyl and methyl alcohols, ether, benzene, toluene, xylene, and acetic acid. It is insoluble in water.

BENZIDINE DERIVATIVE OF 2.5-DIMETHYLBENZALDEHYDE.
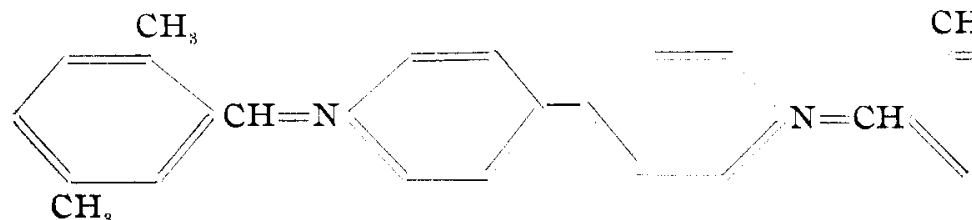

$\mathrm{CH}_{3}$

0.75 gram benzidine were dissolved in 5 grams warm alcohol, 
in a small flask. To this was added I gram 2.5-dimethylbenzaldehyde dissolved in two grams alcohol. The contents of the flask were gently warmed until a yellow crystalline precipitate was formed. This was filtered off by means of a filter-pump, washed well with alcohol, and recrystallized several times out of toluene. The crystals melt at $187.5^{\circ}$ uncorrected temperature, to an opalescent liquid which remains unchanged at $33^{\circ}$. The condensation takes place according to the following equation :
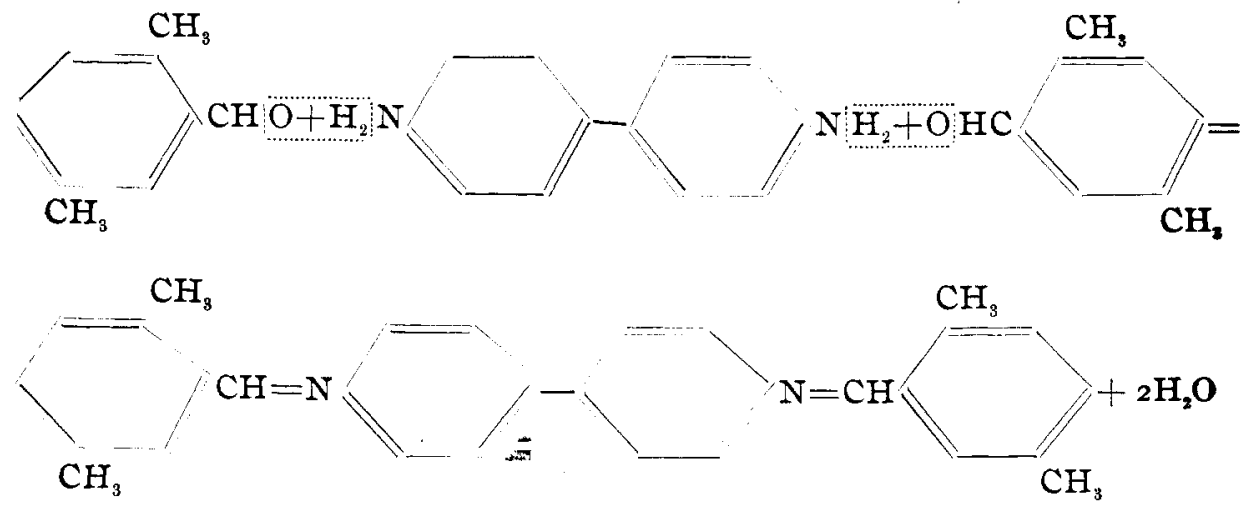

Analysis :

0.08495 gram of the substance burned with cupric oxide gave at $26^{\circ}$ and $746 \mathrm{~mm}$., $5.6 \mathrm{cc}$. nitrogen equivalent to $6.0564 \mathrm{mg}$. nitrogen.

Calculated for
$\mathrm{C}_{30} \mathrm{H}_{98} \mathrm{~N}_{9}(416)$

Nitrogen $\ldots \ldots \ldots \ldots \ldots \ldots \ldots \ldots, 6.73$

Found.

7.12

I. The crystals appear to be orthorhombic. Combination forms are chiefly a pyramid and a pinacoid.

II. The axial ratios are approximately:

$a: b: c=0.5255+: \mathrm{I}:$ approximately $\mathrm{I}+$

III. Axes $b$ and $c$ are very nearly the same length.

Assuming the pinacoid as basal, the forms are

$$
\text { (I) } a: b: c=\text { II } 1 \text {. }
$$

One prism plane was noticed on one crystal.

$$
a: b: \infty c=\mathrm{I} \text { IO. }
$$

IV. The crystal is pleochroic, greenish yellow to yellowish green, showing greatest absorption parallel to axis $b$.

The crystals of the benzidine condensation, which are of a 
sulphur-yellow color and very large, are soluble in benzene, toluene, xylene, chloroform, slightly soluble in acetone and benzine.

They are insoluble in methyl alcohol, ethyl alcohol, ether, hot and cold water.

ANILINE DERIVATIVE OF 2.5-DIMETHYLBENZALDEHYDE.

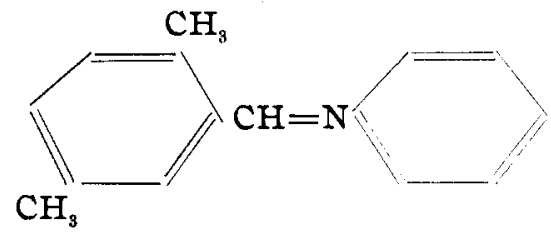

I. 4 grams freshly distilled aniline were placed in a small roundbottomed flask; to this, I gram aldehyde was added, and the mixture heated for three hours on a water-bath. Then the substance, a golden brown liquid, was allowed to stand several days. Upon placing the flask into ice-water, crystals with a yellow tinge separated out. These, recrystallized from not too dilute alcohol, melted at $5 \mathrm{I}^{\circ}$ uncorrected temperature.

If too dilute alcohol is used the condensation product breaks up into its original constituents. The reaction is analogous to that of the benzidine condensation.

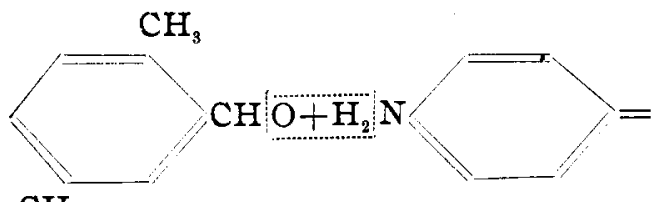

$\mathrm{CH}_{3}$

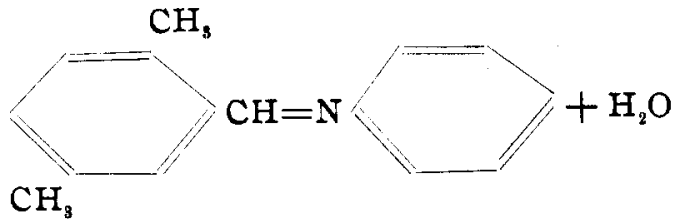

Analysis :

0.0410 gram of the substance burned with cupric oxide at $29^{\circ}$ and $752 \mathrm{~mm}$., gave $2.5 \mathrm{cc}$. nitrogen, corresponding to $2.77 \mathrm{mg}$. nitrogen.
Calculated for
$\mathrm{C}_{15} \mathrm{H}_{15} \mathrm{~N}(209)$.
Nitrogen................... 6.69
Found.
6.78

The crystals form glancing plates very soluble in ether, methyl alcohol, ethyl alcohol, benzene, toluene, xylene, and benzine. 
Water decomposes the compound very easily, forming aniline and aldehyde.

2.5-DIMETHYLCINNAMIC ACID.

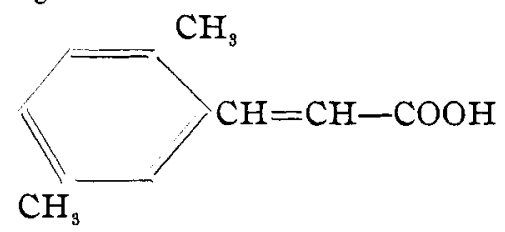

I.25 grams freshly distilled aniline were placed in a round-bottomed flask, and to this I gram redistilled 2.5-dimethylbenzaldehyde was added. The flask was provided with a safety-tube about two feet long, which prevented the entrance of any moisture.

The mixture was boiled three hours on a water-bath; then the temperature was raised a little above $100^{\circ}$.

One gram malonic acid dried at $100^{\circ}$ for one hour was dissolved in absolute alcohol and added to the solution.

The golden brown liquid was allowed to stand over night, the safety tube being plugged to keep out the moisture.

In the morning a light colored crystalline mass formed, which contained no trace of the green color often formed as a byproduct.

In order to remove the aniline set free in the reaction, 3 grams of concentrated hydrochloric acid diluted with 3 grams water were added, and the substance was allowed to stand three-quarters of an hour. At the end of this period, the aniline hydrochloride was filtered off, and the residue washed with water until the washwater remained clear. The residue was then thoroughly extracted with hot benzine. White needles crystallized out of the benzine solution, which melted at $\mathrm{i} 76.5^{\circ}$ uncorrected temperature, when recrystallized out of alcohol.

The yellow substance remaining in the flask was a dye, which dissolved in alcohol to a red-green fluorescent liquid. This was formed as a by-product.

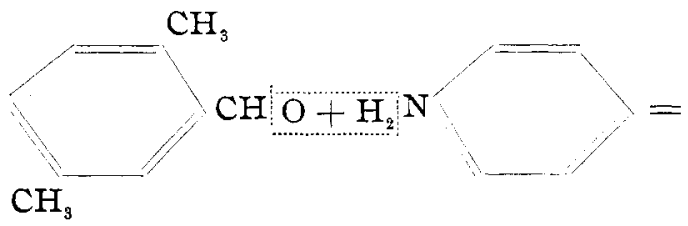



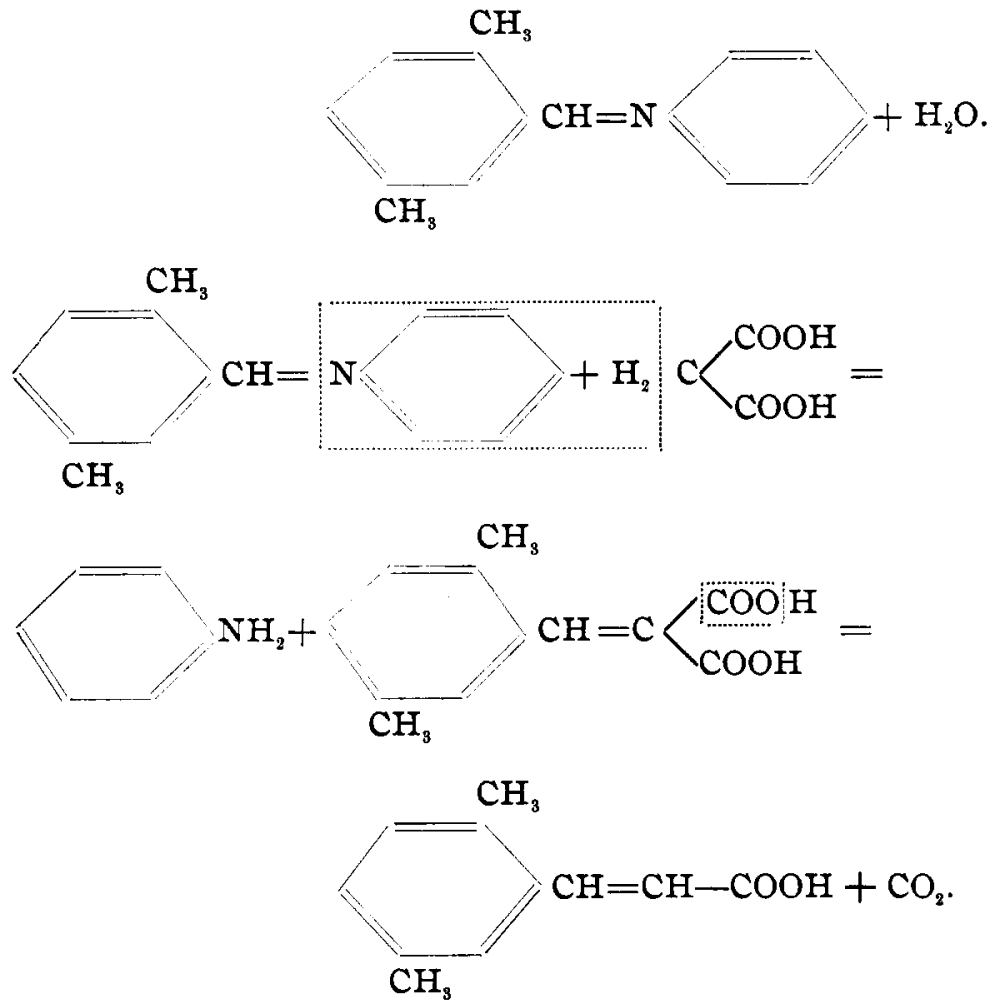

Analysis :

0.1440 gram burned with cupric oxide gave 0.3970 gram carbon dioxide and 0.0872 gram water; equivalent to 0.10827 gram carbon and $0.00968 \mathrm{gram}$ hydrogen.

$$
\begin{aligned}
& \text { Calculated for } \\
& \mathrm{C}_{11} \mathrm{H}_{12} \mathrm{O}_{2}(176) \text {. Found. } \\
& \text { Carbon................... } 75 \\
& \text { Hydrogen } . . . \ldots \ldots \ldots \ldots \ldots \ldots \text { 6.8. } \\
& 74.98 \\
& 6.7 \mathrm{I}
\end{aligned}
$$

The crystals belong to the triclinic system and have indefinite faces. The extinction angle is high.

2.5-Dimethylcinnamic acid is soluble in methyl alcohol, ethyl alcohol, ether, chloroform, benzine, benzene, toluene, xylene, and acetone. It is insoluble in cold water, but soluble in a great excess of hot water.

2.5-Dimethylcinnamic acid acts as an unsaturated compound, forming with bromine a brom compound and with hydrogen a hydrocinnamic acid. 
2.5-DIMETHYLHYDROCINNAMIC ACID.

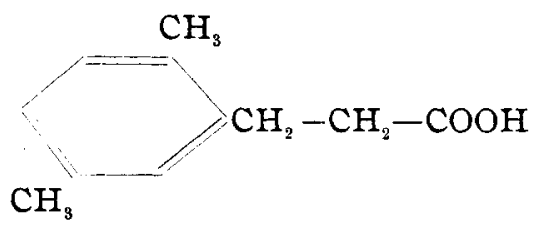

Two grams dimethylcinnamic acid were treated in a small flask with 2 grams water and sufficient dilute sodium hydroxide was added to render the solution clear and weakly alkaline. The sodium salt of the cinnamic acid which is soluble in water was formed.

To form the hydro compound 2 grams of 4 per cent. sodium amalgam were added very gradually in order to utilize all the hydrogen generated. When all the mercury had been set free the contents of the flask were gently warmed on the water-bath, and then the mercury was filtered off.

Dilute hydrochloric acid was added to the filtrate until all the acid was separated out as a white precipitate. This was filtered, pressed, and dried on a porous plate, and crystallized twice from benzene. The needle-shaped crystals melt at I I $1.5^{\circ}$ uncorrected temperature.

The reduction takes place in the following manner :

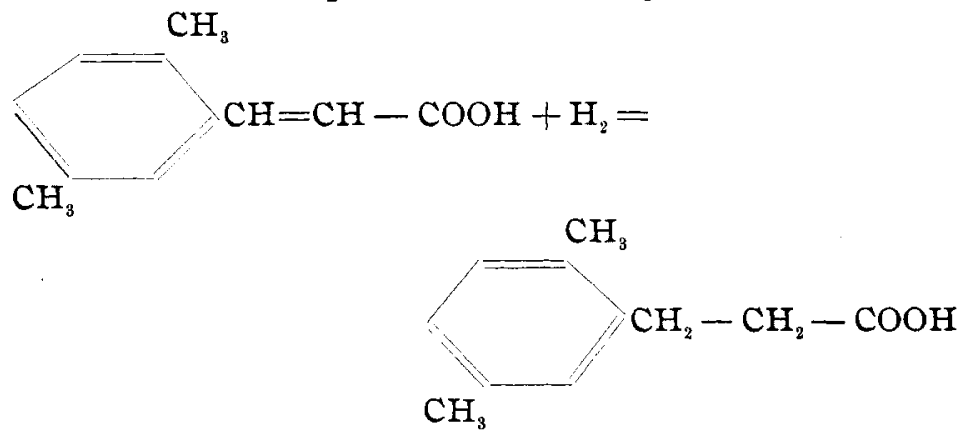

TRITHIO DERIVATIVE OF 2.5-DIMETHYLBENZALDEHYDE.
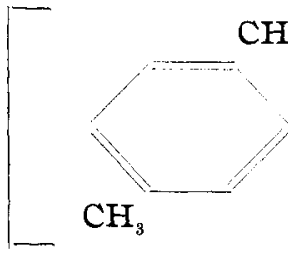

CHS 
One gram 2.5-dimethylbenzaldehyde was dissolved in 7.5 grams absolute alcohol. Three grams of concentrated hydrochloric acid were added to this solution. A rapid stream of hydrogen sulphide was passed through this mixture three and a half hours at room temperature.

The solution first turned blue, due to formation of the monothio derivative, then a white precipitate separated out, and at the end of the reaction a pinkish yellow adhesive mass mixed with a predominant amount of a chalk-like precipitate had formed.

The supernatant liquid was poured off, the residue thoroughly washed with alcohol and then pressed and dried on a porous plate.

The substance dissolved in benzene would not crystallize. A crystal of iodine was then added to the solution, which was then allowed to stand several weeks, when large colorless plates of the monoclinic system separated out. These were recrystallized from absolute alcohol.

Melting-point $=110^{\circ}$ uncorrected temperature. The thio derivative is a polymer.

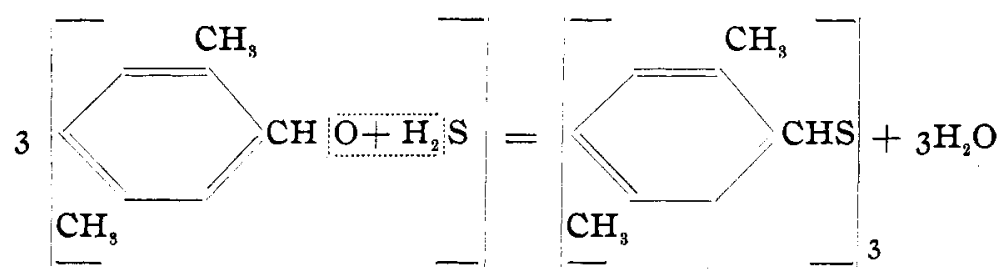

Owing to the small amount of the pure substance obtained, no combustion was made, but the molecular weight was determined by the depression of the freezing-point.

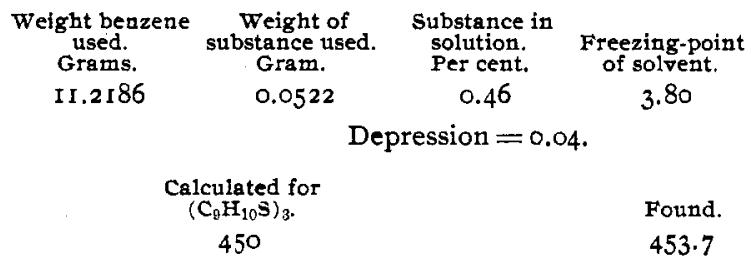
of solvent.

3.80

Depression $=0.04$.

Calculated for

$\left(\mathrm{C}_{9} \mathrm{H}_{10} \mathrm{~S}\right)_{3}$.

Found.

$45^{\circ}$ of substance.

The crystals are soluble in benzene, absolute alcohol, chloroform and insoluble in water. They possess the peculiar characteristic odor of the aromatic thioaldehydes. 